\title{
Genome-wide identification and analysis of cystatin family genes in Sorghum (Sorghum bicolor L.)
}

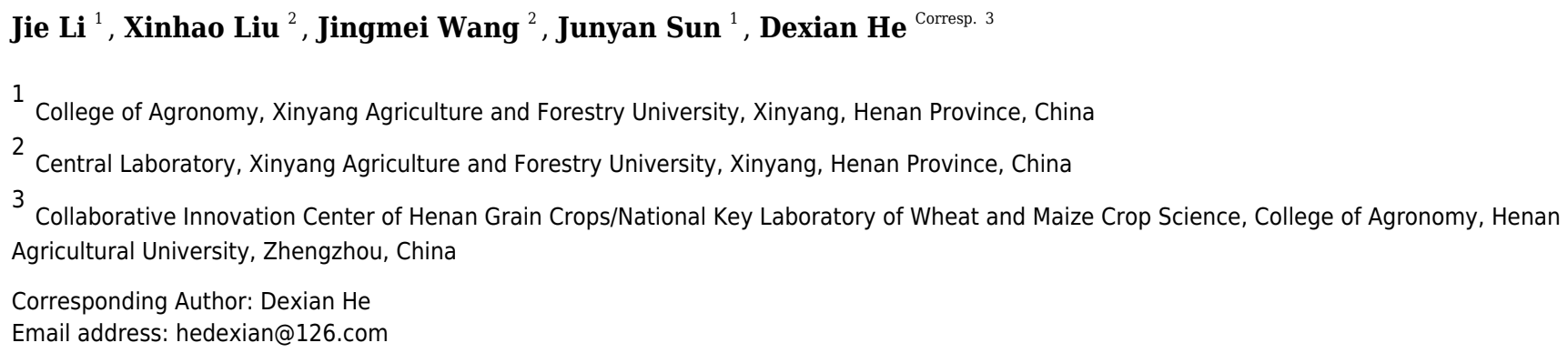

To set a systematic study of the Sorghum cystatins ( $\mathrm{SbCys}$ ) gene family, a comprehensive genome-wide analysis of the SbCys family genes was performed by bioinformatics-based methods. In total, 18 SbCys genes were identified in Sorghum, which were distributed unevenly on chromosomes, and two genes were involved in a tandem duplication event. All SbCys genes had similar exon/intron structure and motifs, indicating their high evolutionary conservation. Transcriptome analysis showed that 16 SbCys genes were expressed in different tissues, and most genes displayed higher expression levels in reproductive tissues than in vegetative tissues, indicating that the SbCys genes participated in the regulation of seed formation. Furthermore, the expression profiles of the SbCys genes revealed that 7 cystatin family genes were induced during Bipolaris sorghicola infection and only 2 genes were responsive to aphid infestation. In addition, quantitative real-time polymerase chain reaction (qRT-PCR) confirmed that 17 SbCys genes were induced by one or two abiotic stresses (dehydration, salt, and ABA stresses). The interaction network indicated that SbCys proteins were associated with several biological processes, including seed development and stress responses. Notably, the expression of SbCys4 was up-regulated under biotic and abiotic stresses, suggesting its potential roles in mediating the responses of Sorghum to adverse environmental impact. Our results provide new insights into the structural and functional characteristics of the SbCys gene family, which lay the foundation for better understanding the roles and regulatory mechanism of Sorghum cystatins in seed development and responses to different stress conditions. 


\section{Genome-wide identification and analysis of cystatin family genes}

2

3

4

\section{Author affiliation:}

61 College of Agronomy, Xinyang Agriculture and Forestry University, Xinyang, Henan 464001, 7 China

82 Central Laboratory, Xinyang Agriculture and Forestry University, Xinyang, Henan 464001, 9 China

103 Collaborative Innovation Center of Henan Grain Crops/National Key Laboratory of Wheat and

\section{in Sorghum (Sorghum bicolor L. Moench)}

Jie $\mathrm{Li}^{1}$, Xinhao Liu ${ }^{2}$, Jingmei Wang ${ }^{2}$, Junyan Sun ${ }^{1}$, Dexian $\mathrm{He}^{3 *}$ 


\section{ABSTRACT}

To set a systematic study of the Sorghum cystatins (SbCys) gene family, a comprehensive genome-wide analysis of the $S b C y s$ family genes was performed by bioinformatics-based methods. In total, 18 SbCys genes were identified in Sorghum, which were distributed unevenly on chromosomes, and two genes were involved in a tandem duplication event. All SbCys genes had similar exon/intron structure and motifs, indicating their high evolutionary conservation. Transcriptome analysis showed that $16 S b C y s$ genes were expressed in different tissues, and most genes displayed higher expression levels in reproductive tissues than in vegetative tissues, indicating that the SbCys genes participated in the regulation of seed formation. Furthermore, the expression profiles of the SbCys genes revealed that 7 cystatin family genes were induced during Bipolaris sorghicola infection and only 2 genes were responsive to aphid infestation. In addition, quantitative real-time polymerase chain reaction (qRT-PCR) confirmed that $17 S b C y s$ genes were induced by one or two abiotic stresses (dehydration, salt, and ABA stresses). The interaction network indicated that SbCys proteins were associated with several biological processes, including seed development and stress responses. Notably, the expression of SbCys4 was up-regulated under biotic and abiotic stresses, suggesting its potential roles in mediating the responses of Sorghum to adverse environmental impact. Our results provide new insights into the structural and functional characteristics of the $S b C y s$ gene family, which lay the foundation for better understanding the roles and regulatory mechanism of Sorghum cystatins in seed development and responses to different stress conditions.

\section{INTRODUCTION}

Cystatins are competitive and reversible inhibitors of cystein proteases from families $\mathrm{C} 1 \mathrm{~A}$ and 
46 C13, which have been identified in many plant species (Martinez and Diaz, 2008; Zhao et al.

47 2014). Cystatins are categorized into three groups, including stefins without disulfide bonds

48 (Group I), cystatins with four conserved Cys residues forming two disulfide bonds (Group II), and kininogens with repeated, stefin-like domains (Group III) (Meriem et al. 2010). Cystatins are widely distributed in animal and plant systems (Tremblay et al. 2019). Based on their primary sequence homology, cystatins contain three signature motifs including a $\mathrm{QxVxG}$ reactive site, a tryptophan residue (W) located downstream of the reactive site, and one or two glycine (G) residues in the flexible $\mathrm{N}$ terminus of the protein. These three motifs are important for the cystatin inhibitory mechanism (Martinez et al. 2009). In addition, a consensus sequence ([LVI][AGT]-[RKE]-[FY]-[AS]-[VI]-x-[EDQV]-[HYFQ]-N) in cystatins is conformed to a predicted secondary $\alpha$-helix structure (Meriem et al. 2010). Most plant cystatins are small proteins with a molecular mass in the $12-$ to $16-\mathrm{kD}$ range (Meriem et al. 2010). Some plant cystatins contain a C-terminal extension that raises their molecular weights up to $23 \mathrm{kDa}$. The longer $\mathrm{C}$-terminal extensions are thought to be involved in the inhibition of cysteine protease activities in the peptidase C13 family (Martinez et al. 2007; Martinez and Diaz, 2008).

The principal functions of plant cystatins are related to the regulation of endogenous cystein proteases during plant growth and development, senescence, and programmed cell death (Belenghi et al. 2010; Díazmendoza et al. 2014; Zhao et al. 2014). Additionally, plant cystatins have been used as effective molecules against different pests and pathogens (Martinez et al. 2016). For example, several publications reported the inhibition of recombinant cystatins on the growth of some pests and fungi (Lima et al. 2015; Tremblay et al. 2019). Tomato plants overexpressing the wheat cystatin TaMDC1 displayed a broad stress resistance to bacterial pathogen, and the defense responses were mediated by methyl jasmonate and salicylic acid (Christova et al. 
2018). The inhibition of amaranth cystatin on the digestive insect cysteine endopeptidases was observed by Valdés-Rodríguez et al. (2015). Plant cystatins are also involved in the responses to abiotic stresses, such as over-expression of MpCYS4 in apple delayed natural and stress-induced leaf senescence (Tan et al. 2017). Song et al. (2017) found that the expression of AtCYS5 was induced by heat stress (HS) and exogenous ABA treatment in germinating seed, furthermore, over expression of AtCYS5 enhanced HS tolerance in transgenic Arabidopsis.

To date, cystatin family genes had been well described in several plant species such as Arabidopsis, rice, soybean, wheat, Populus trichocarpa, and Brachypodium distachyon (Martinez and Diaz, 2008; Wang et al. 2015; Yuan et al. 2016; Dutt et al. 2016; Subburaj et al. 2017). However, a genome-wide study of cystatin family genes in Sorghum has not yet been performed. Sorghum is the world's fifth biggest crop (after rice, wheat, maize, and barley), belonging to a $\mathrm{C} 4$ grass that grows in arid and semi-arid regions (Taylor et al. 2010). Its drought tolerance is a consequence of morphological and anatomical characteristics (i.e., thick leaf wax, deep root system) and physiological responses (i.e., stay-green, osmotic adjustment). Hence, Sorghum is an excellent model plant for the study of plant response to drought stress (Sunita et al. 2011). Recently, the completion of the whole genome assembly of Sorghum (Sorghum bicolor L. Moench) makes it possible to identify and analyze cystatin family genes in Sorghum (Paterson et al. 2009). In this study, we aimed to perform a genome-wide identification of $S b C y s$ family genes in Sorghum and analyze their phylogeny, conserved motifs, structure, cis-elements, and expression profile in different tissues. We also explored the expression patterns of $S b C y s$ genes in response to biotic and abiotic stresses. The results may lay a foundation for further functional analyses of cystatin genes. 
92 MATERIALS AND METHODS

93 Identification of SbCys family members in Sorghum genome

94 The identification of SbCys candidates was conducted according to the methods of Lozano et al.

95 (2015) with some modification. The cystatin sequences of Arabidopsis, rice (Oryza sativa), and

96 barely (Hordeum vulgare) were downloaded from TAIR (http://www.Arabidopsis.org), the Rice

97 Genome Annotation Project (http://rice.plantbiology.msu.edu/index.shtml), and Ensembl

98 database (http://plants.ensembl.org/Hordeum vulgare/Info/Index), respectively. The whole-

99 genome sequence of Sorghum was downloaded from Ensembl database

100 (http://plants.ensembl.org/Sorghum_bicolor/Info/Index). Then predicted proteins from the

101 Sorghum genome were scanned using HMMER v3 (http://hmmer.org/) using the Hidden Markov

102 Model (HMM) profile of cystatin (PF00031) from the Pfam protein family database

103 (http://pfam.xfam.org/) (Finn et al. 2011). From the proteins obtained using the raw cystatin

104 HMM, a high-quality protein set with a cut-off $e$-value $<1 \times 10^{-10}$ was aligned and used to 105 construct a Sorghum specific cystatin HMM using hmmbuild from the HMMER v3 suite. Then

106 all proteins with $e$-value $<0.01$ were selected by the new Sorghum specific HMM. Cystatin

107 sequences were further filtered based on the closest homolog from Arabidopsis, Oryza sativa, 108 and Hordeum vulgare using ClustalW and the UNIREF100 sequence database. Proteins that 109 have no typical domain (Aspartic acid proteinase inhibitor) and reactive site motif (QxVxG) 110 were removed from posterior analysis.

\section{Sequence alignment, structure analysis, and phylogenetic tree construction}

112 The Multiple Expectation for Motif Elicitation (MEME) program was used to identify conserved 113 motifs shared among SbCys proteins. The parameters of MEME were as follows: maximum 114 number of motifs, 10; optimum width, between 6 and 50; and number of repetitions, any. 
115 The three-dimensional structures of Sorghum cystatins were modelled by the automated SWISS-

116 MODEL program (http://swissmodel.expasy.org/interactive). The known crystal structure of rice

117 oryzacystatin I (OC-I) and SiCYS (Hu et al. 2015; Yuan et al. 2016) were used to construct the

118 homology-based models. Structure analysis was conducted by the RasMol 2.7 program.

119 A phylogenetic tree was constructed using MEGA X with the maximum likelihood method 120 according to the Whelan and Goldman + freq. Model. Bootstrap analysis was performed by 1000 121 replicates with the p-distance model. The phylogenetic tree was visualized and optimized in 122 Figtree (http://tree.bio.ed.ac.uk/software/figtree/).

123 Transcript structures, chromosomal location and gene duplication

124 The genomic structure of each SbCys gene was derived from the alignment of their coding 125 sequence to their corresponding genome full-length sequence. The diagrams of these $S b C y s$ 126 genes were drawn by the Gene Structure Display Server (GSDS, http://gsds.cbi.-pku.edu.cn/) 127 (Hu et al. 2014). The chromosomal locations of SbCys genes were retrieved from the 128 Sorghum_bicolor_NCBIv3 map. The genes were plotted on chromosomes using the Map 129 Gene2chromosome (MG2C, version 2.0) tool (http://mg2c.iask.in/). Gene duplication events of $130 S b C y s$ family genes were investigated according to the following two criteria: (1) the alignment 131 covered $>75 \%$ of the longer gene, (2) the aligned region had an identity $>75 \%$, (3) located in 132 less than $100 \mathrm{~kb}$ single region or separated by less than five genes. For microsynteny analysis, 133 the CDS sequence of every cystatin from Arabidopsis, barley, rice, and Sorghum was used as the 134 query to search against all other cystatins using NCBI_blast software with $e$-value $\leq 1 \mathrm{e}^{-10}$. The 135 Circos software was used to display the results of collinear gene pairs (Krzywinski et al. 2009).

\section{Calculation of Ka and Ks}

137 To assess the degree of natural selection on $S b C y s$ genes, the rate ratio of $K a$ (nonsynonymous 
138 substitution rate) to $K s$ (synonymous substitution rate) was calculated using KaKs Calculator 2.0

139 (Zhang et al. 2006). The $\mathrm{Ka} / \mathrm{Ks}$ ratio $>1,<1$, or $=1$ indicates positive, negative, or neutral

140 evolution, respectively (Yadav et al. 2015).

\section{Promoter analysis of $S b C y s$ genes}

142 To investigate the cis-regulatory elements in a promoter region, the upstream sequences $(1.5 \mathrm{~kb})$ 143 of the start codon in each SbCys gene were scanned in the PlantCARE database 144 (http://bioinformatics.psb.ugent.be/webtools/plantcare/html/) and New PLACE 145 (https://www.dna.affrc.go.jp/PLACE/?action=newplace).

146 Analysis of interaction networks of the SbCys proteins

147 The functional interacting network models of SbCys proteins were integrated using the web 148 STRING program (http://string-db.org/) based on an Arabidopsis association model; the 149 confidence parameters were set at a 0.40 threshold, the number of interactors was set to five 150 interactors. Arabidopsis AtCys proteins were mapped to Sorghum SbCys proteins based on their 151 homologous relationship. The interaction network of SbCys proteins was drawn by 152 Cytoscape_v3.6.0.

\section{Expression analysis of $\boldsymbol{S b}$ Cys genes under biotic stresses}

154 The RNA-Seq data used for investigating the expression patterns of SbCys genes in various 155 tissues were downloaded from the NCBI SRA (Sequence Read Archive) database (ERP024508) 156 (Wang et al. 2018). Root, shoot, and seedling were collected at 14 days after germination. 157 Embryo, endosperm, and pericarp were collected at 20 days after pollination. Pollen samples 158 were collected at booting stage. Inflorescences were collected according to the sizes: 1-5 mm, 5$15910 \mathrm{~mm}$, and 1-2 cm. Three biological replicates were performed for each plant tissue. RNA was 160 sequenced using the Illumina HiSeq 2500 system to generate $250 \mathrm{bp}$ pair-end reads. 
161 RNA-seq data of biotic stresses were obtained from two experiments. The first experiment 162 measured the transcriptome response of a resistant Sorghum (Sorghum bicolor L. Moench) 163 infected with Bipolaris sorghicola (Yazawa et al. 2013). RNA samples were collected at 0, 12, 164 and 24 hours post-inoculation with one biological replicate. RNA-seq was run using Illumina 165 technology to give 100-base-pair single-end reads on a HiSeq2000 system. The second study 166 measured changes in the transcriptome of Sorghum leaves infested by sugarcane aphid (Tetreault 167 et al. 2019). The RNA-seq data were downloaded from the NCBI SRA database. In this study, 168 two treatments (infested and control) were arranged and two Sorghum genotypes (resistant 169 cultivar RTx2783 and susceptible cultivar BCK60) were used. Leaf samples were collected from treated and control plants at 5, 10, and 15 days post sugarcane aphid infestation. Three biological

171 replicates were performed for all treatment and time combinations. RNA was sequenced using 172 the Illumina Hiseq 2500 platform to generate $100 \mathrm{bp}$ single end reads. The accession numbers 173 and sample information were listed in Table S1. The differential expression of $S b C y s$ genes were 174 investigated by Hisat2 (http:/kim-lab.org/), Htseq (http://www.htseq.org/), and DESeq2 (R 175 package) based on the RNA-seq data (Wen, 2017). The $p \leq 0.05$ and $|\operatorname{logFC}| \geq 1.5$ were set as the 176 cut-off criterion.

\section{Plant materials and treatments}

178 Seed of Sorghum (Sorghum bicolor L. cv. Jinza 35) were surface sterilized (15 min in 4\% $179 \mathrm{NaClO}$ ), washed with distilled water several times, and transferred to moist germination paper 180 for 3 days in an incubator at $25^{\circ} \mathrm{C}$. These seedlings were grown in holes of foam floating plastic containers (30 seedlings per container) with constant aeration in Hoagland solution in a growth room with $14 \mathrm{~h} / 30{ }^{\circ} \mathrm{C}$ light and $10 \mathrm{~h} / 22{ }^{\circ} \mathrm{C}$ dark regime. The nutrient solution was routinely 183 changed every 3 days. At the three-leaf stage (the juvenile phase (Hashimoto et al. 2019)), 
184 abiotic stresses including ABA, salinity, and dehydration treatments were initiated according to 185 the procedures described in previous reports (Dugas et al. 2011; Wang et al. 2012; Yan et al. 2017). The plants were transferred quickly to the nutrient solution containing $0.1 \mathrm{mM} \mathrm{ABA}$ (dissolved in ethanol), $5 \mu \mathrm{L}$ ethanol (control for ABA treatment), $250 \mathrm{mM}$ sodium chloride $(\mathrm{NaCl})$, or $15 \%(\mathrm{~W} / \mathrm{V})$ polyethylene glycol (PEG) 6,000. The central part of flag leaves from randomly selected Sorghum plants were harvested respectively at 0,12 , and 24 hours posttreatment per trial, and immediately frozen in liquid nitrogen and then stored at $-80{ }^{\circ} \mathrm{C}$ prior to RNA isolation. For each treatment at a given time, three biological replicates were used. The leaf samples of 10 plants came from the same container for one biological replicate. That is, three containers were used for three biological replicates respectively.

\section{RNA extraction and $q R T-P C R$ analysis}

Total RNA of $100 \mathrm{mg}$ leaf samples was isolated using the "TaKaRa MiniBEST Plant RNA Extraction" Kit (TaKaRa, Dalian, China) following the manufacturer's instructions. Purity and concentration of RNA samples were evaluated by measuring the $A_{260} / A_{230}$ and $A_{260} / A_{280}$ ratios. In order to digest the genomic DNA, the RNAs were treated with RNase-free DNase I. Reverse transcription was performed according to the kit instructions (Promega, Madison, USA). Primer pairs for qRT-PCR analysis were designed by Primer3Plus program (http://www.bioinformatics.nl), and were shown in Table S2. A $20 \mu$ reaction volume containing $0.4 \mu \mathrm{l}$ of each primer (forward and reverse), $2 \mu \mathrm{l}$ 10-fold diluted cDNA, $7.2 \mu \mathrm{l}$ of nuclease-free water, and $10 \mu \mathrm{l}$ of GoTaq ${ }^{\circledR}$ qPCR Master Mix (Perfect Real Time; Promega). PCR reaction included one cycle at $95{ }^{\circ} \mathrm{C}$ for 3 min, followed by 39 cycles of $95{ }^{\circ} \mathrm{C}$ for $15 \mathrm{~s}, 60{ }^{\circ} \mathrm{C}$ for $30 \mathrm{~s}$, and $72{ }^{\circ} \mathrm{C}$ for 20s. The reactions were conducted using the CFX96 Real-Time PCR Detection System (Bio-Rad Laboratories, Inc.). Three independent biological replicates and two technical 
207 replicates of each sample were performed. Gene-specific amplification of both reference and 208 cystatin genes were standardized by the presence of a single, dominant peak in the qRT-PCR 209 dissociation curve analyses. All data were analyzed by CFX Manager Software (Bio-Rad 210 Laboratories, Inc.). The efficiency range of the qRT-PCR amplifications for all of the genes 211 tested was between 91\% and 100\%. The average target (SbCys) cT (threshold cycle) values were 212 normalized to reference $(\beta$-actin) $\mathrm{cT}$ values. The fold change between treated sample and control 213 was calculated using the slightly modified $2^{-(\Delta \Delta \mathrm{Ct})}$ method as described by Kebrom et al. (2010). 214 A probability of $p \leq 0.05$ was considered to be significant.

\section{RESULTS}

\section{Identification and analysis of $S b C y s$ genes}

218 To extensively identify all of SbCys family members in Sorghum, we constructed a Sorghum219 specific HMM for the SbCys domain to scan the Sorghum genome, and 22 gene candidates were 220 identified. After removing the repetitive and/or incomplete sequences, the rest of SbCys 221 sequences were submitted to Pfam (http://pfam.xfam.org/) and SMART (http://smart.embl222 heidelberg.de/) to confirm the conserved domain. Finally, a total of 18 non-redundant SbCys proteins were identified and were serially renamed from $S b C y s 1$ to $S b C y s 17$ according to their

224 location and order in chromosomes. Gene names, gene IDs, chromosomal locations, amino acid numbers, protein sequences, and annotations assigned to GO terms of the identified SbCys proteins were listed in Table S3. The average length of these SbCys proteins was 148 amino acid residues and the length mainly centered on the range of 105 to 240 amino acid residues. 
230 by chromosomes 9 and 3 (4 and 3 genes, respectively). Chromosomes 2 and 4 had just one

231 SbCys gene, whereas chromosomes 5, 6, 7, 8, and 10 had no SbCys genes.

232 Gene structure analysis of $\operatorname{SbCys}$ genes

233 The analysis of exon-intron structure can provide useful information about the gene function, 234 organization, and evolution of multiple gene families ( $\mathrm{Xu}$ et al. 2012). Schematic structures of $235 S b C y s$ genes from Sorghum were obtained using the GSDS program (Fig. 2). Among the $S b C y s$ 236 genes, more than half $(12,66.7 \%)$ were intronless, three genes (SbCys 11, SbCys 15, and $S b C y s 16)$ 237 had one intron, two genes (SbCys14 and SbCys17) had two introns, and one gene (SbCys10) had 238 three introns. These six $S b C y s$ genes with one or more introns were clustered into one clade, suggesting the evolutionary event may affect the gene structure (Altenhoff et al. 2012).

240 Sequence alignment, protein motifs analysis, and structural predication of SbCys

241 Alignments of SbCys sequences were carried out to search for amino acid variants that could 242 lead to differences in their inhibitory capability for cysteine proteases. The results were shown in 243 Fig. 3a. N-terminal and C-terminal extensions with varying lengths that presented in several 244 SbCys proteins were not displayed in the comparison. These predicted structures shared many 245 identical residues including $\alpha$-helix and the four $\beta$-sheets ( $\beta 2-5$ ) (Fig. 3a). Analysis of conserved motifs of SbCys proteins also revealed that some typical conserved motifs could be detected in most SbCys proteins, such as motif 1, 2, 3, and 4. These motifs formed a fundamental structural combination (Fig. 3b and 3c). Motif 1 was conserved in the central loop region with a consensus sequence of "QxVxG" and could be detected in most SbCys proteins, which played an important role in the inhibitory capacity of cystatins towards their target cysteine proteases (Meriem et al. 2010). Motif 2 contained a particular consensus sequence ([LVI][GA][RQG][WF]AV) that conformed to a predicted secondary $\alpha$-helix structure (Martinez et al. 2009). The other two 
253 typical motifs for SbCys proteins, motif 3 (V[WY][EVG]KPW) and motif 4 ([RK]xLxxF),

254 which were firstly described in tobacco (Zhao et al. 2014), were also detected in most SbCys

255 proteins, indicating their conserved and common role in both dicots and monocots. Motif 5

256 existed only in 3 SbCys family members (SbCys5, SbCys8, and SbCys15). Details of the 5

257 conserved motifs were shown in Fig. S1.

258 The predicted three-dimensional structures of the Sorghum cystatins were established using the

259 SWISS-MODEL program based on the known crystal structure of OC-I and SiCYS (Fig. 4).

260 Although these structures were predicted with variable degrees of accuracy, all of Sorghum

261 cystatins shared similar protein structure with rice OC-I (Fig. 4a), excepting SbCys10 that shared

262 similar protein structure with SiCYS (Fig. 4b). In additon, SbCys14 showed a significant

263 variation in its predicted three-dimensional structures, might due to an extra $\alpha$-helix that existed

264 in the C-terminal extension of SbCys14. Two important motifs (the conserve QxVxG motif and

265 W residue) of Sorghum cystatins involved in the interaction with the target cysteine enzymes

266 were also shown in Fig. 4. The predicted structure of SbCys13 showed some distortions in the

267 region of the $\beta 2$ sheet, probably due to the insertion of a methionine in the first position of the

268 conserved QxVxG motif.

269 Phylogenetic analysis of SbCys genes

270 The cystatin gene family is highly conserved in both monocots and dicotyledons (Martinez and 271 Diaz, 2008). To investigate the phylogenic relationships of SbCys proteins to other known plant 272 cystatins, a multiple sequence alignment of SbCys sequences to the sequences from Arabidopsis, 273 rice, and barley was conducted by the ClustalW program. As showed in Fig. 5, these cystatins 274 were categorized into three groups, including Group I, Group II, and Group III. A total of 21 275 cystatins were classified to Group I and 6 cystatins from Sorghum. Group II contained 7 
276 cystatins, only one cystatin from Sorghum. The remaining 21 proteins were assigned to Group III

277 and 11 SbCys proteins fell into this group. In addition, some bootstrap values in the phylogenetic

278 tree were low, suggesting that high sequence differentiation in these cystatins occurred.

279 Microsynteny analysis indicated that one orthologous gene pair was identified in the cross of 280 barley and Sorghum, rice and Sorghum, respectively, while no orthologous gene pair between 281 Arabidopsis and Sorghum was found (Fig. S2). These data indicated that SbCys genes were more 282 closely related to rice and barley than Arabidopsis. Interestingly, a pair of SbCys genes (SbCys22831 and SbCys2-2) was involved in the tandem duplication event in Sorghum (Fig. S2). Analysis of 284 duplicated SbCys genes showed that the $K a / K s$ ratio far less than 1, varying from 0.0976 to 2850.5679 (Table S4), indicating that negative selection occurred in the duplication event.

\section{Promoter analysis of $\boldsymbol{S b} \boldsymbol{C} \boldsymbol{y s}$ genes}

287 In order to obtain useful information on the regulatory mechanism of cystatin gene expression, the $1.5 \mathrm{~kb}$ upstream sequences from the translation start sites of $S b C y s$ genes were submitted into PlantCARE database to detect the cis-elements. Various putative plant regulatory elements in the promoter region of SbCys genes were shown in Fig. 6 and Table S5. Several potential regulatory elements involved in stress-related transcription factor-binding sites were found, including Gbox, W-box, TC-rich repeats, MBS, heat shock elements (HSEs), and ABA-response element (ABRE). The identified SbCys genes possessed at least 1 stress-response-related cis-element, suggesting that the expressions of $S b C y s$ genes were related to the biotic and abiotic stresses. All of $S b C y s$ genes had one or more G-box with the exception of $S b C y s 9$, implying that these $S b C y s$ genes could be induced by light stress. 14 SbCys genes possessed MBS element, ABRE element was found in 12 SbCys genes, HSE element was located in 10 SbCys genes, and TC-rich repeats and $\mathrm{W}$-boxes were located in 8 genes. In addition Skn-1_motif was conserved in the promoter 
299 regions of most $S b C y s$ genes, indicating these genes were associated with the regulation of seed 300 storage protein gene expression (Strömvik and Fauteux, 2009). The high diversity of the cis301 acting elements suggested that these SbCys genes might have a wide range of functional roles 302 and could be involved in multiple stress responses and growth and development progress (Zhang 303 et al. 2008).

\section{Protein interaction network of SbCys proteins}

305 In this study, the interactions of the SbCys proteins were investigated in an Arabidopsis 306 association model using STRING software. As shown in Fig. 7, the interaction network of cystatins showed a complex functional relationship. AtCys2 (corresponding to SbCys12) interacted with stress related proteins (AT1G56280, AT3G19580, AT5G67450, and AtCys1) and growth and development related proteins (AT1G63100 and AT5G04340), AtCys1 (corresponding to SbCys11, 15, 16, and 17) interacted with some vacuolar-processing enzyme which involved in processing of vacuolar seed protein precursors into the mature forms, and AtCys5 (corresponding to SbCys1, 2-1, 3, 4, 5, 6, 7, 8, 9, and 13) interacted with several lipidtransfer proteins (AT1G07747, AT1G52415, AT2G16592, AT3G29152, and AT4G12825). The results suggested that cystatins might be associated with many biological processes by protein interactions, such as pollen development, stress responses, and seed maturation (Wang et al. 316 2012).

\section{Expression profile of $S b C y s$ genes in different Sorghum tissues}

318 To obtain the spatial and temporal expression patterns of all SbCys genes, RNA-seq data 319 (ERP024508) were downloaded to explore the expression levels of SbCys genes in different tissues including root, stem, seedling, pollen, endosperm, embryo, inflorescence (1-5mm, 1- 
322 in one tissue at least, except for $S b C y s 13$, which were barely expressed in any tissue. The

323 expression patterns of $S b C y s$ genes were significantly different between reproductive tissues and

324 vegetative tissues, such as $S b C y s 2-1, S b C y s 3, S b C y s 4, S b C y s 5, S b C y s 7, S b C y s 9, S b C y s 12$, and

325 SbCys 17, which showed relatively higher expression levels in reproductive tissues including

326 pollen, endosperm, embryo, and pericarp than in vegetative tissues, while the expression of

$327 S b C y s 7$ and $S b C y s 15$ were higher in vegetative tissues than in reproductive tissues. It was worth

328 noting that the majority of $S b C y s$ genes had lower expression levels during inflorescence

329 development excepting $S b C y s 17$ which displayed a higher expression pattern.

330 Expression of $S b C y s$ genes under biotic stresses

331 To gain insight into the potential roles of $S b C y s$ genes in response to Bipolaris sorghicola 332 infection and sugarcane aphid infestation, the relative expression patterns of these genes were 333 investigated by using the public transcription data from NCBI SRA database (DRP000986 and 334 SRP162227, respectively). As shown in Fig. 9 and 10, the expression patterns of $S b C y s$ genes 335 were different under the two biotic stresses. In response to Bipolaris sorghicola infection, seven $336 S b C y s$ genes were induced and only 2 genes (SbCys12 and $S b C y s 13)$ were suppressed in the 337 infected Sorghum leaves compared with control (Fig. 9a). However, under aphid infestation, four $338 S b C y s$ genes $(S b C y s 4, S b C y s 10, S b C y s 11$, and $S b C y s 14)$ were up-regulated and 3 genes (SbCys1, $339 S b C y s 3$, and $S b C y s$ 17) were down-regulated relative to control in the susceptible Sorghum line 340 (BCK60). In the resistant Sorghum line (RTx2783), only two SbCys genes (SbCys4 and SbCys11) 341 were induced, and the rest were barely expressed in Sorghum leaves with aphid infestation (Fig. $3429 \mathrm{~b}$ and 10). These results might suggest that $S b C y s$ genes played different roles in responding to 343 pathogen infection and aphid infestation.

\section{Expression profiling of $S b C y s$ genes under abiotic stresses}


345 We also investigated the expression of $S b C y s$ genes in response to various abiotic stresses 346 including dehydration, salt shock, and ABA (Fig. 11). Under dehydration stress, seven $S b C y s$ 347 genes (SbCys4, SbCys5, SbCys6, SbCys9, SbCys10, SbCys11, and $S b C y s 17)$ were induced to 348 present a significant up-regulation from 0 to $24 \mathrm{~h}$, while the expressions of $S b C y s 2-1, S b C y s 12$, $349 S b C y s 15$, and SbCys 16 were decreased. Furthermore, the expressions of 4 SbCys genes (SbCys 1 , $350 S b C y s 3, S b C y s 8$, and $S b C y s 14$ ) displayed an up-down trend from $0 \mathrm{~h}$ to $24 \mathrm{~h}$ (Fig. 11a). With salt 351 shock treatment, the expressions of SbCys2-1, SbCys3, SbCys4, SbCys8, SbCys10, and SbCys11 352 were significantly up-regulated at all treatment time points, whereas SbCys16 showed a 353 significant down-regulated trend (Fig. 11b). In addition, SbCys6, SbCys13 SbCys14, SbCys15, and $S b C y s 17$ showed up-down expression trends, but $S b C y s 5$ displayed a down-up expression pattern (Fig. 11b). After exogenous ABA treatment, the expressions of $4 S b C y s$ genes (SbCys2-2, $S b C y s 3, S b C y s 4$, and $S b C y s 7)$ were significantly up-regulated at three time points, but 9 genes (SbCys1, SbCys2-1, SbCys5, SbCys8, SbCys10, SbCys11, SbCys13, SbCys14, and SbCys17) were down-regulated. Additionally, $S b C y s 12, S b C y s 15$, and $S b C y s 16$ displayed up-down expression trends (Fig. 11c). Interestingly, all $S b C y s$ genes were up-regulated in response to one or two stresses except $S b C y s 4$ that was significantly induced under dehydration, salt, and ABA stresses, suggesting that SbCys4 might play an important role in response to different stress responses.

\section{DISCUSSION}

Plant cystatins are a group of intrinsic small proteins, whose members play important roles in diverse biological processes and stress responses (Martinez et al. 2016; Meriem et al. 2010).

Recently, a large number of sequence data from different plant species have been uploaded in GenBank, which provide convenience for us to describe their characteristics, and several 
368 cystatins families have been identified from plants, such as rice, soybean, and wheat (Wang et al. 369 2015; Dutt et al. 2016; Yuan et al. 2016). However, little is known about the cystatin family in 370 Sorghum. In the present study, we identified 18 SbCys genes from the Sorghum genome. The 371 number was less than that of $B$. distachyon genome, where $25 B d C y s$ members were identified 372 (Subburaj et al. 2017). The 18 members in Sorghum was a larger number than found in rice (11 373 genes) and Arabidopsis (7 genes) (Wang et al. 2015), but was similar to soybean (20 members) 374 (Yuan et al. 2016). The difference on the cystatin number might reflect the adaptation of plants 375 to environment.

376 The identified SbCys genes were unevenly distributed on chromosomes 1, 2, 3, 4, and 9, and half 377 of them were distributed on chromosome 1 (Fig. 1). The uneven distribution of cystatin genes in 378 chromosomes was also found in the $B$. distachyon genome and the Oryza sativa genome 379 (Subburaj et al. 2017; Wang et al. 2015). This phenomenon might be due to the tandem 380 duplication events of cystatin genes on the chromosomes (Li et al. 2017). Several tandem 381 duplication events occurred at chromosomes 1 of the B. distachyon genome (Subburaj et al. 382 2017). Two tandem duplication events (OsCys4/OsCys5 and OsCys6/OsCys7) were found 383 among OsCys genes, and existed in chromosomes 1 and 3 (Wang et al. 2015). One tandem duplication event (SbCys2-1/SbCys2-2) occurred at chromosome 1 of the Sorghum genome (Fig. S2). Eighteen SbCys genes were divided into three groups based on phylogenetic analysis (Fig. 5). Some conserved motifs among SbCys proteins had been identified by the alignment of the amino acid sequences (Fig. 3). However, the conservation was accompanied with the differences in some important amino acids indicated that SbCys family members might undergo a complex evolutionary history. The variation of crucial amino acids of cystatins might have a significant 390 influence on their respective functions (Tremblay et al. 2019). For example, the QxVxG motif 
391 could directly enter and interact with the active site of targeted enzymes. The motif was

392 conserved in all SbCys proteins with the exceptions of 5 cystatins (SbCys1, SbCys6, SbCys8,

393 SbCys9, and SbCys13) that were partially modified by the insertion or variation in important

394 residues (Fig. 3a). Furthermore, three SbCys proteins (SbCys8, SbCys9, and SbCys 13) showed

395 significant variations with other Sorghum cystatins in their predicted three-dimensional

396 structures (Fig. 4). The variations in vital amino acid residues might result in the change in

397 cystatin inhibitory action (Tremblay et al. 2019). In addition, two novel motifs, motif 3

398 (V[WY][EVG]KPW) and motif 4 ([RK]xLxxF), firstly described in tobacco (Zhao et al. 2014),

399 were also identified in the C-terminalin of many SbCys proteins. The contribution of the two

400 new motifs to cystatin inhibitory action needs to be further studied.

401 During past decades, plant cystatins were reported to play essential roles in inhibiting 402 endogenous and exogenous cysteine proteases activities during seed development (Tremblay et 403 al. 2019). In the present study, as revealed by RNA-seq data analysis (Fig. 8 and S3), the 404 expression levels of several SbCys family genes were higher in reproductive tissues than in 405 vegetative tissues, which were consistent with the reports that most cystatins were specifically 406 expressed in developing seeds and played a role in seed development (Dutt et al. 2010; Zhao et al. 407 2014). Moreover, promoter analysis showed that the highly expressed SbCys genes in 408 reproductive tissues possessed endosperm expression-related cis-elements (Skn-1 and 409 GCN4_motif) (Fig. 6 and Table S5). Our protein interaction prediction results also showed that 410 several SbCys proteins could interact with many functional proteins (e.g., growth and 411 development related proteins, vacuolar-processing enzyme, and lipid-transfer proteins) (Fig. 7), 412 implying these cystatins were involved in regulating the gene expression of cereal grain storage 413 proteins (Diaz-Mendoza et al. 2016). 
414 Plant cystatins are involved in various biotic stress responses and probably act as defense

415 proteins against pest infestation and pathogen infection (Meriem et al. 2010). At present, some

416 cystatins with insecticidal activity have been isolated from many plants, such as barley, tomato,

417 and potato (Rasoolizadeh et al. 2017; Siddiqui et al. 2017; Velasco-Arroyo et al. 2018; Goulet et

418 al. 2020). Several cystatins having antifungal activities were also isolated from taro, cacao, and

419 wheat (Christova et al. 2018; Pirovani et al. 2010; Chen et al. 2014). Although studies on

420 insecticidal and antifungal activity of plant cystatins have been well established in vitro, the

421 knowledge about their roles in plants in response to biotic stresses is limited. To explore the

422 properties of $S b C y s$ genes responding to pest infestation and pathogen infection, we conducted

423 the analysis on the expression patterns of $S b C y s$ genes. The results showed that the expressions

424 of most SbCys genes were induced during Bipolaris sorghicola infection, suggesting these

425 cystatins played functions in inhibiting exogenous cysteine proteases secreted by pathogens to

426 infect plant tissues (Fig. 9a). Interestingly, for sugarcane arthropods infestation, only two genes

427 (SbCys4 and SbCys 11) were up-regulated significantly in susceptible and resistant Sorghum lines

428 (Fig. 9b and 10). These differential expression patterns between SbCys genes might suggest that

429 some of them had evolved to inhibit specific cysteine proteinases. The exact roles of these $S b C y s$

430 genes in insecticidal and antifungal activity in vivo are worthy to be explored in further study.

431 Cystatin genes are also involved in various abiotic stress responses in plants. In Arabidopsis, the 432 expression levels of $A t C Y S 1$ and $A t C Y S 2$ were enhanced by high temperature and wounding 433 stresses (Hwang et al. 2010). AtCYSa and $A t C Y S b$ were also induced by different abiotic stresses, 434 e.g., salt, drought, oxidation, and cold stresses (Zhang et al. 2008). Velasco-Arroyo et al. (2018) 435 reported that the silence of barley $H v C P I-2$ and $H v C P I-4$ specifically modified leaf responses to 436 drought stress. Wang et al. (2015) observed the significant change in the expression levels of 
437 several rice $O s C Y S$ genes under cold, drought, salt, and hormone treatments. In the present study,

438 most SbCys genes were found to have positive or negative responses to dehydration, salt, and

439 ABA stresses. Moreover, the interaction results showed that most cystatins could interact with

440 stresses-related proteins, implying that the cystatins played critical roles in response to diverse

441 stress conditions. Notably, the expression of SbCys4 was significantly up-regulated under three

442 stress conditions (Fig. 11), suggesting a specific role of SbCys4 in responding to various stress

443 conditions. Promoter analysis indicated that stress-related cis-elements were widespread in the

444 promoter region of these cystatin genes (Table S5), and SbCys4 possessed plenty of stress-related 445 cis-elements, including G-box, ABRE, HSE, MBS, and TC-rich repeats. These results provide an 446 effective reference for the functional verification of the $S b C y s$ family genes under abiotic 447 stresses.

449 CONCLUSIONS

450 In the current study, we identified 18 SbCys family genes in the Sorghum genome through a 451 genome-wide survey. The chromosomal localization, conserved protein domain, gene structure, 452 phylogenetic relationship, as well as the interaction network of these $S b C y s$ genes was 453 systematically analyzed, revealing special characteristics of $S b C y s$ family genes in Sorghum. The 454 identified SbCys genes displayed an uneven distribution in Sorghum chromosomes. All SbCys 455 genes shared similar exon/intron organization and conserved motifs. Phylogenetic analysis 456 suggested that Sorghum cystatins had higher homology with monocotyledon than dicotyledon. 457 Furthermore, the variation of amino acids in Sorghum cystatin critical active sites suggested that 458 they might undergo a complex evolutionary process and possess structural and functional 459 divergence. The expression profiles of $S b C y s$ genes in different tissues indicated that most $S b C y s$ 
460 genes were involved in plant growth and development. Changes in the expression of $S b C y s$

461 genes under biotic and abiotic stresses indicated that many $S b C y s$ genes played important roles in

462 response to unfavorable growth conditions. It was worth noting that the expression of $\operatorname{SbCys} 4$

463 was significantly enhanced under biotic and abiotic stresses, suggesting its unique role in 464 mediating the response of Sorghum to adverse environmental conditions.

465

466

467

468

469

470

471

472

473

474

475

476

477

478

479

480

481

482

\section{REFERENCES}

Altenhoff AM, Studer RA, Robinsonrechavi M, Dessimoz C. 2012. Resolving the ortholog conjecture: orthologs tend to be weakly, but significantly, more similar in function than $\begin{array}{lllll}\text { paralogs. } & \text { PLoS } & \text { Computational Biology } & \text { DOI } 1002514\end{array}$ 10.1371/journal.pcbi.1002514.

Belenghi B, Acconcia F, Trovato M, Perazzolli M, Bocedi A, Polticelli F, Ascenzi P, Delledonne M. 2010. AtCYS1, a cystatin from Arabidopsis thaliana, suppresses hypersensitive cell death. European Journal of Biochemistry 270(12):2593-604 DOI 10.1046/j.1432-1033.2003.03630.x.

Blanca VA, Mercedes DM, Andrea GS, Santamaria B, Estrella M, Miguel TB, Kumlehn G, Martinez J, Diaz I. 2018. Silencing barley cystatins $H v C P I-2$ and $H v C P I-4$ specifically modifies leaf responses to drought stress. Plant Cell Environment 41:1776-1790 DOI $10.1111 /$ pce. 13178 .

Chen PJ, Senthilkumar R, Jane WN, He Y, Tian Z, Yeh KW. 2014. Transplastomic Nicotiana benthamiana plants expressing multiple defence genes encoding protease inhibitors and chitinase display broad-spectrum resistance against insects, pathogens, and abiotic stresses. Plant Biotechnology Journal 12(4):1-13 DOI 10.1111/pbi.12157. 
483

484

485

486

487

488

489

490

491

492

493

494

495

496

497

498

499

500

501

502

503

504

505

Christova PK, Christov NK, Mladenov PV, Imai R. 2018. The wheat multidomain cystatin TaMDC1 displays antifungal, antibacterial, and insecticidal activities in planta. Plant Cell Reports 37:923-932 DOI 10.1007/s00299-018-2279-4.

Diaz-Mendoza M, Dominguez-Figueroa JD, Velasco-Arroyo B, Cambra I, GonzalezMelendi P, Lopez-Gonzalvez A, Garcia A, Hensel G, Kumlehn J, Diaz I, Martinez M. 2016. HvPap-1 C1A protease and HvCPI-2 cystatin contribute to barley grain filling and germination. Plant Physiology 170:2511-2524. DOI 10.1104/pp.15.01944.

Díazmendoza M, Velascoarroyo B, Gonzálezmelendi P, Martínez M, Díaz I. 2014. C1A cysteine protease-cystatin interactions in leaf senescence. Journal of Experimental Botany 65(14):3825-33 DOI 10.1093/jxb/eru043.

Dugas DV, Monaco MK, Olson A, Klein RR, Kumari S, Ware D, Klein PE. 2011. Functional annotation of the transcriptome of Sorghum bicolor in response to osmotic stress and abscisic acid. BMC Genomics 12:514 DOI 10.1186/1471-2164-12-514.

Dutt S, Singh VK, Marla SS, Kumar A. 2010. In silico analysis of sequential, structural, and functional diversity of wheat cystatins and its implication in plant defense. Genomics Proteomics Bioinformatics 8(1):42-56 DOI 10.1016/S1672-0229(10)60005-8.

Finn RD, Clements J, Eddy SR. 2011. HMMER web server: interactive sequence similarity searching. Nucleic Acids Research 39:29-37 DOI 10.1093/nar/gkr367.

Goulet MC, Sainsbury F, Michaud D. 2020. Cystatin activity-based protease profiling to select protease inhibitors useful in plant protection. Methods in Molecular Biology 2139:353-366 DOI 10.1007/978-1-0716-0528-8.

Hashimoto S, Tezuka T, Yokoi S. 2019. Morphological changes during juvenile-to-adult phase transition in Sorghum. Planta 250:1557-1566 DOI 10.1007/s00425-013-1895-z. 
506 Hu B, Jin J, Guo AY, Zhang H, Luo J, Gao G. 2014. GSDS 2.0: an upgraded gene feature 507 visualization server. Bioinformatics 31(8): 1296 DOI 10.1093/bioinformatics/btu817.

508

509

510

511

512

513

514

515

516

517

518

519

520

521

522

523

524

525

526

527

528

Hu YJ, Irene D, Lo CJ, Cai YL, Tzen TC, Lin TH, Chyan CL. 2015. Resonance assignments and secondary structure of a phytocystatin from Sesamum indicum. Biomolecular NMR Assignments 9:309-311 DOI 10.1007/s12104-015-9598-y.

Hwang JE, Hong JK, Lim CJ, Chen H, Je J, Yang KA, Kim DY, Choi YJ, Lee SY, Lim CO. 2010. Distinct expression patterns of two Arabidopsis phytocystatin genes, AtCYS1 and AtCYS2, during development and abiotic stresses. Plant Cell Reports 29:905-915 DOI 10.1007/s00299-010-0876-y.

Kebrom TH, Brutnell TP, Finlayson SA. 2010. Suppression of sorghum axillary bud outgrowth by shade, phyB and defoliation signalling pathways. Plant Cell Environment 33(1):48-58 DOI 10.4161/psb.5.3.11186.

Kiggundu A, Muchwezi J, Van C, Viljoen A, Vorster J, Schlüter U, Kunert K, Michaud D. 2010. Deleterious effects of plant cystatins against the banana weevil Cosmopolites sordidus. Arch Insect Biochemistry Physiology 73(2):87-105 DOI 10.1002/arch.20342.

Kiyosaki T, Matsumoto I, Asakura T, Funaki J, Kuroda M, Misaka T, Arai S, Abe K. 2007. Gliadain, a gibberellin-inducible cysteine proteinase occurring in germinating seeds of wheat, Triticum aestivum L., specifically digests gliadin and is regulated by intrinsic cystatins. FEBS Journal 164:470-477 DOI 10.1111/j.1742-4658.2007.05749.x.

Krzywinski M, Schein J, Birol I, Connors J, Gascoyne R, Horsman D, Jones SJ, Marra MA. 2009. Circos: an information aesthetic for comparative genomics. Genome research 19(9):1639-1645 DOI 10.1101/gr.092759.109.

Li J, Yang XW, Li YC, Niu JS, He DX. 2017. Proteomic analysis of developing wheat grains 
529

530

531

532

533

534

535

536

537

538

539

540

541

542

543

544

545

546

547

548

549

550

551

infected by powdery mildew (Blumeria graminis f.sp. tritici). Journal of Plant Physiology 215:140-153 DOI 10.1016/j.jplph.2017.06.003.

Li SF, Su T, Cheng GQ, Wang BX, Li X, Deng CL, Gao WJ. 2017. Chromosome evolution in connection with repetitive sequences and epigenetics in plants. Genes 8:290 DOI 10.3390/genes 8100290 .

Lima AM, dos Reis SP, de Souza CR. 2015. Phytocystatins and their potential to control plant diseases caused by fungi. Protein and Peptied Letters 22:104-111 DOI $10.2174 / 0929866521666140418101711$.

Lozano R, Hamblin MT, Prochnik S, Jannink JL. 2015. Identification and distribution of the NBS-LRR gene family in the Cassava genome. BMC Genomics 16(1):360 DOI 10.1186/s12864-015-1554-9.

Martinez M, Cambra I, Carrillo L, Diazmendoza M, Diaz I. 2009. Characterization of the entire cystatin gene family in barley and their target cathepsin L-like cysteine-proteases, partners in the hordein mobilization during seed germination. Plant Physiology 151(3):1531-1545 DOI 10.1104/pp.109.146019.

Martinez M, Diazmendoza M, Carrillo L, Diaz I. 2007. Carboxy terminal extended phytocystatins are bifunctional inhibitors of papain and legumain cysteine proteinases. FEBS Letters 581(16):2914-2918 DOI 10.1016/j.febslet.2007.05.042.

Martinez M, Diaz I. 2008. The origin and evolution of plant cystatins and their target cysteine proteinases indicate a complex functional relationship. BMC Evolutionary Biology 8(1):198-210 DOI 10.1186/1471-2148-8-198.

Martinez M, Santamaria ME, Diazmendoza M, Arnaiz A, Carrillo L, Ortego F, Diaz I. 2016. Phytocystatins: defense proteins against phytophagous insects and acari. 
552

553

554

555

556

557

558

559

560

561

562

563

564

565

566

567

568

569

570

571

572

573

574

International Journal of Molecular Sciences 17(10):1747-1763 DOI 10.3390/ijms17101747.

Meriem B, Urte S, Juan V, Marie-Claire G, Dominique M. 2010. Plant cystatins. Biochimie 92(11):1657-1666 DOI 10.1016/j.biochi.2010.06.006.

Paterson AH, Bowers JE, Bruggmann R, Dubchak I, Grimwood J, Gundlach H, Haberer G, Hellsten U, Mitros T, Poliakov A. 2009. The Sorghum bicolor genome and the diversification of grasses. Nature 457(7229):551-556 DOI 10.1038/nature07723.

Pfaffl MW. 2001. A new mathematical model for relative quantification in real-time RT-PCR. Nucleic Acids Research 29:e45 DOI 10.1093/nar/29.9.e45.

Pirovani CP, Santiago AS, Santos LS, Micheli F, Margis R, Silva Gesteira R, Alvim FC, Pereira GAG, Mattos JC. 2010. Theobroma cacao cystatins impair Moniliophthora perniciosa mycelial growth and are involved in postponing cell death symptoms. Planta 232(6):1485-1497 DOI 10.2307/23391912.

Rasoolizadeh A, Goulet MC, Guay JF, Cloutier C, Michaud D. 2017. Population-associated heterogeneity of the digestive Cys protease complement in Colorado potato beetle, Leptinotarsa decemlineata. Journal of Insect Physiology 106:125-133 DOI 10.1016/j.jinsphys.2017.03.001.

Siddiqui AA, Khaki PS, Bano B. 2017. Interaction of almond cystatin with pesticides: Structural and functional analysis. Journal Molecular Recognitnition 30(3):e2586 DOI I 10.1002/jmr.2586.

Song C, Kim T, Chung WS, Lim CO. 2017. The Arabidopsis phytocystatin AtCYS5 enhances seed germination and seedling growth under heat stress conditions. Molecular Cells 40(8):577-586 DOI 10.14348/molcells.2017.0075. 
575 Strömvik MV, Fauteux F. 2009. Seed storage protein gene promoters contain conserved DNA 576 motifs in Brassicaceae, Fabaceae, and Poaceae. BMC Plant Biology 9:126 DOI $10.1186 / 1471-2229-9-126$.

578

579

580

581

582

583

584

585

586

587

588

589

590

591

592

593

594

595

596

597

Subburaj S, Zhu D, Li X, Hu Y, Yan Y. 2017. Molecular characterization and expression profiling of Brachypodium distachyon L. cystatin genes reveal high evolutionary conservation and functional divergence in response to abiotic stress. Frontiers in Plant Science 8:743 DOI 10.3389/fpls.2017.00743.

Sunita K, Klein RR, Andrew O, Monaco MK, Dugas DV, Doreen W, Klein PE. 2011. Functional annotation of the transcriptome of Sorghum bicolor in response to osmotic stress and abscisic acid. BMC Genomics 12(1):514-514 DOI 10.1186/1471-2164-12-514.

Tan Y, Yang Y, Li C, Liang B, Li M, Ma F. 2017. Overexpression of $M p C Y S 4$, a phytocystatin gene from Malus prunifolia (Willd.) Borkh., delays natural and stress-induced leaf senescence in apple. Plant Physiology Biochemistry 115:219-28 DOI 10.1016/j.plaphy.2017.03.025.

Taylor SH, Hulme SP, Rees M, Ripley BS, Woodward FI, Osborne CP. 2010. Ecophysiological traits in $\mathrm{C}_{3}$ and $\mathrm{C}_{4}$ grasses: A phylogenetically controlled screening experiment. New Phytologist 185(3):780-791 DOI 10.1111/j.1469-8137.2009.03102.x.

Tetreault HM, Grover S, Scully ED, Gries T, Palmer N, Sarath G, Louis J, Sattler SE. 2019. Global responses of resistant and susceptible Sorghum (Sorghum bicolor) to sugarcane aphid (Melanaphis sacchari). Frontiers in Plant Science 10:145 DOI 10.3389/fpls.2019.00145.

Tremblay J, Goulet MC, Michaud D. 2019. Recombinant cystatins in plants. Biochimie 166:184-193 DOI 10.1016/j.biochi.2019.06.006. 
598 Valdes-Rodriguez S, Galvan-Ramirez JP, Guerrero-Rangel A, Cedro-Tanda A. 2015. 599 Multifunctional amaranth cystatin inhibits endogenous and digestive insect cysteine 600 endopeptidases: A potential tool to prevent proteolysis and for the control of insect pests. 601 Biotechnology Applied Biochemistry 62:634-641 DOI 10.1002/bab.1313.

602

603

604

605

606

607

608

609

610

611

612

613

614

615

616

617

618

619

620

Velasco-Arroyo B, Diaz-Mendoza M, Gomez-Sanchez A, Moreno-Garcia B, Santamaria ME, Torija-Bonilla M, Hensel G, Kumlehn J, Martinez M, Diaz I 2018. Silencing barley cystatins HvCPI-2 and HvCPI-4 specifically modifies leaf responses to drought stress. Plant Cell and Environment 41(8):1776-1790 DOI 10.1111/pce.13178.

Wang B, Regulsk M, Tseng E, Olson A, Goodwin S, McCombie WR, Ware D. 2018. A comparative transcriptional landscape of maize and Sorghum obtained by singlemolecule sequencing. Genome Research 28(6):921-928 DOI 10.1101/gr.227462.117.

Wang HW, Hwang SG, Karuppanapandian T, Liu AH, Kim W, Jang CS. 2012. Insight into the molecular evolution of non-specific lipid transfer proteins via comparative analysis between rice and sorghum. DNA Research 19:179-194 DOI 10.1093/dnares/dss003.

Wang W, Zhao P, Zhou XM, Xiong HX, Sun MX. 2015. Genome-wide identification and characterization of cystatin family genes in rice (Oryza sativa L.). Plant Cell Reports 34(9):1579-1592 DOI 10.1007/s00299-015-1810-0.

Wen G. 2017. A simple process of RNA-Sequence analyses by Hisat2, Htseq, and DESeq2. International Conference Pp:11-15 DOI 10.1145/3143344.3143354.

Xu G, Guo C, Shan H, Kong H. 2012. Divergence of duplicate genes in exon-intron structure. PNAS 109(4):1187-1192 DOI 10.1073/pnas.1109047109.

Yadav CB, Bonthala VS, Muthamilarasan M, Pandey G, Khan Y, Prasad M. 2015. Genome-wide development of transposable elements-based markers in foxtail millet and 
621

622

623

624

625

626

627

628

629

630

631

632

633

634

635

636

637

638

639

640

641

642

643

construction of an integrated database. DNA Research 22:79-90 DOI 10.1093/dnares/dsu039.

Yan S, Li SJ, Zhai GW, Lu P, Deng H, Zhu S, Huang RL, Shao JF, Tao YZ, Zou GH. 2017. Molecular cloning and expression analysis of duplicated polyphenol oxidase genes reveal their functional differentiations in Sorghum. Plant Science 263:23-30 DOI 10.1016/j.plantsci.2017.07.002.

Yazawa T, Kawahigashi H, Matsumoto T, Mizuno H. 2013. Simultaneous transcriptome analysis of Sorghum and Bipolaris sorghicola by using RNA-seq in combination with De novo transcriptome assembly. PLoS One 8(4):e62460 DOI 10.1371/journal.pone.0062460.

Yuan S, Li R, Wang L, Chen H, Zhang C, Chen L, Hao Q, Shan Z, Zhang X, Chen S. 2016. Search for nodulation and nodule development-related cystatin genes in the genome of soybean (Glycine max). Frontiers in Plant Science 7:1595 DOI 10.3389/fpls.2016.01595.

Zhang X, Liu S, Takano T. 2008. Two cysteine proteinase inhibitors from Arabidopsis thaliana, AtCYSa and AtCYSb, increasing the salt, drought, oxidation, and cold tolerance. Plant Molecular Biology 68:131-143 DOI 10.1007/s11103-008-9357-x.

Zhao P, Zhou XM, Zou J, Wang W, Wang L, Peng XB, Sun MX. 2014. Comprehensive analysis of cystatin family genes suggests their putative functions in sexual reproduction, embryogenesis, and seed formation. Journal of Experimental Botany 65(17):5093-5108 DOI $10.1093 / \mathrm{jxb} / \mathrm{eru} 274$.

Zhang Z, Li J, Zhao XQ, Wang J, Wong GK, Yu J. 2006. KaKs_Calculator 2.0: Calculating $\mathrm{Ka}$ and $\mathrm{Ks}$ through model selection and model averaging. Genomics Proteomics Bioinformatics 4(4):259-263 DOI 10.1016/S1672-0229(10)60008-3. 
Figure 1

Chromosome localization of SbCys genes.

Chromosome number is indicated at the top of each bar. The size of chromosome was labeled on the left of the figure.

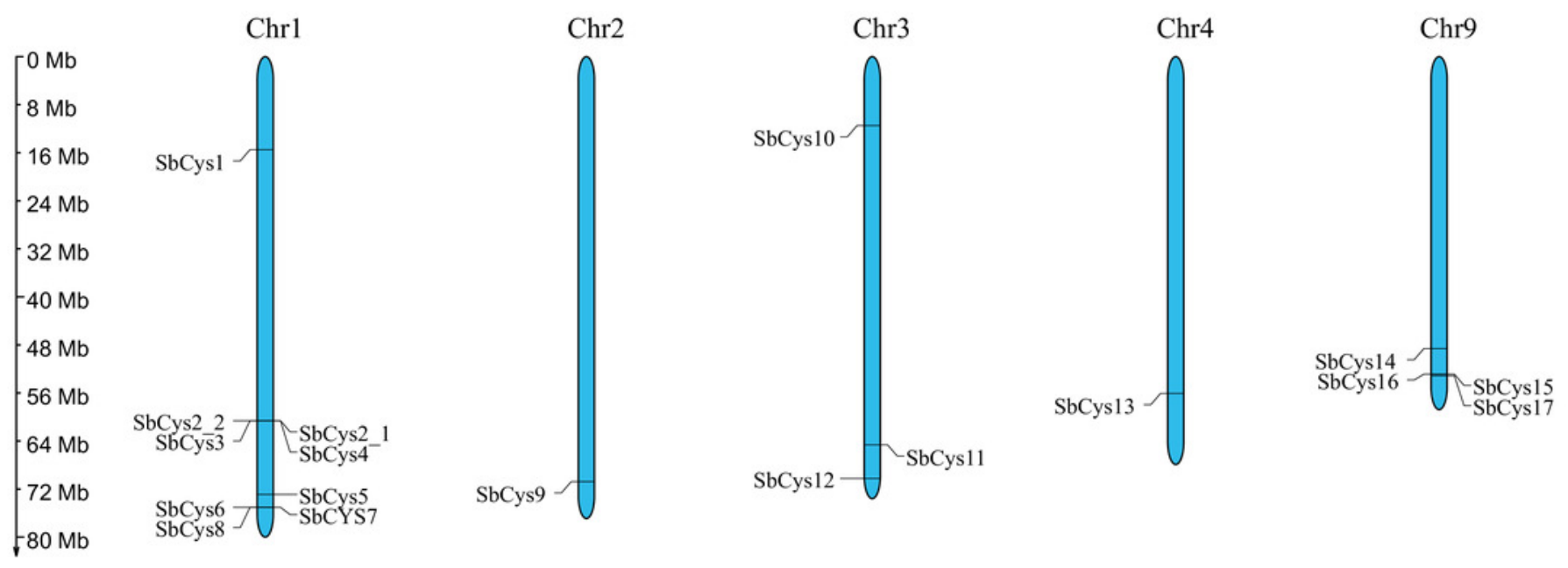


Figure 2

Phylogenetic relationship and gene structure of SbCys genes.

A phylogenetic tree was constructed using MEGA $X$ by the maximum likelihood method with 1000 bootstrap replicates. Exon/intron structures were identified by online tool GSDS.

Lengths of exons and introns of each SbCys genes were exhibited proportionally. Exons and introns are shown by blue bars and black horizontal lines, respectively.

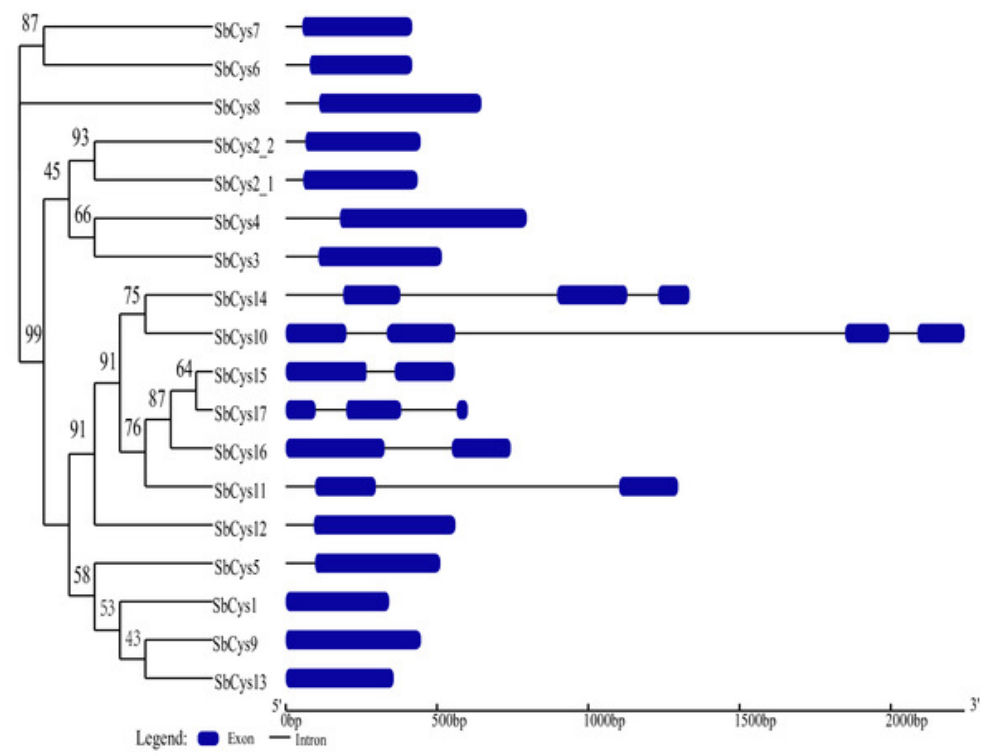


Figure 3

The amino acid alignment and conserved motifs distribution of SbCys.

(A) The locations of the secondary structures ( $\alpha$-helix and $\beta$-sheets) were included. The main cystatin conserved motifs are in black boxes. The strong and weak conservative changes in amino acids are marked by dark gray and light gray font, respectively. (B) The motifs were identified by MEME. Each motif was represented by one color box. (C) Conserved protein motif 1 (QxVxG), motif 2 ( LARFAV and G-residue), motif 3 (W-residue), motif 4 ([RK]xLxxF), and motif 5(P-residue) presented in the variable region of cystatin genes.

A
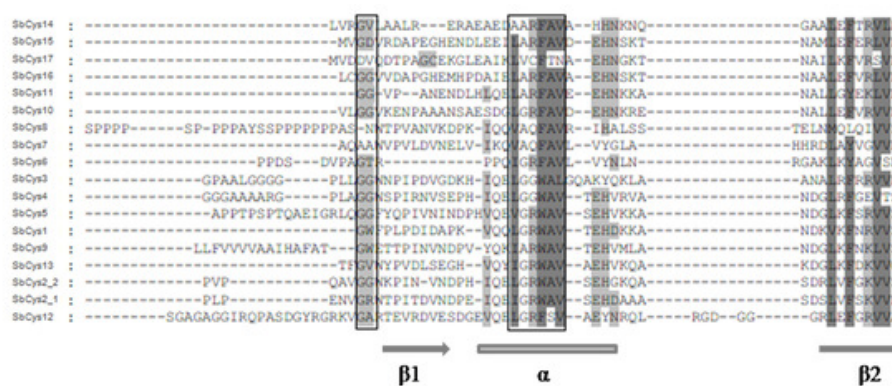

B

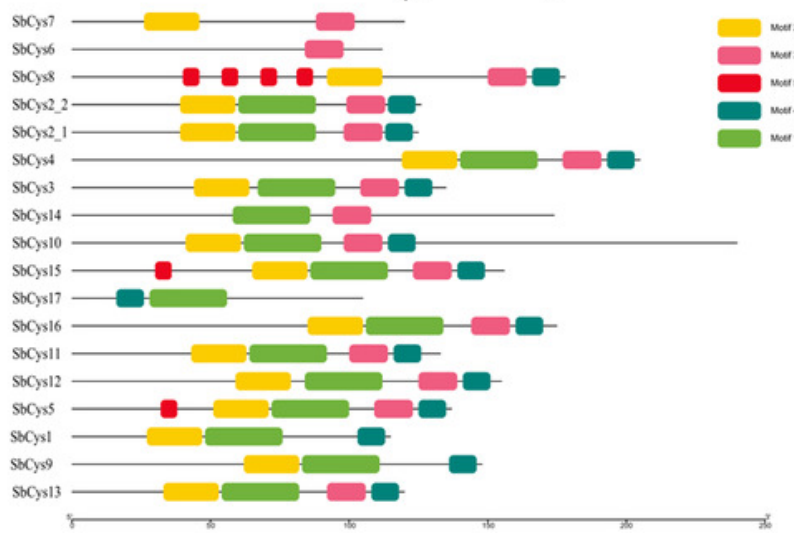

$\mathrm{C}$

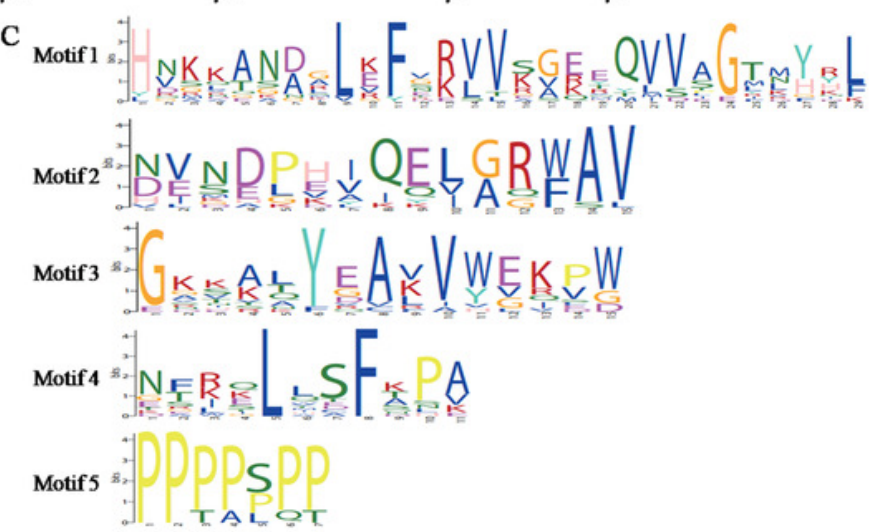


Figure 4

The three-dimensional structure prediction of Sorghum cystatins.

(A) The three-dimensional structures of SbCys proteins were predicted using the automated SWISS-MODEL program with OC-I as a template. (B) The three-dimensional structure of SbCys10 was predicted using the automated SWISS-MODEL program with SiCYS as a template. Two important motifs involved in the interaction with the target enzymes are indicated: the reactive site (asterisks) and W residue (crosses).

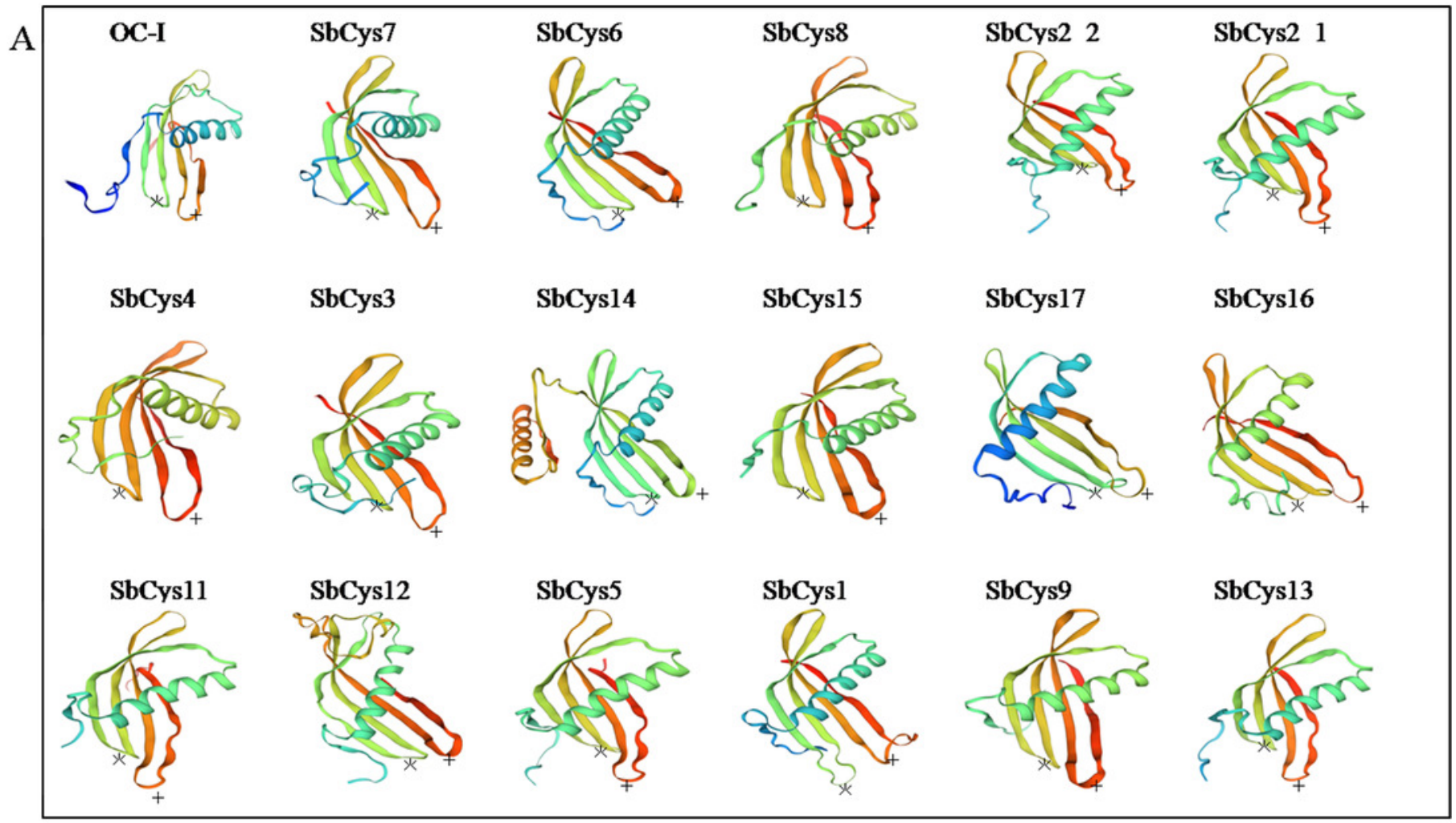

$\mathrm{B}$

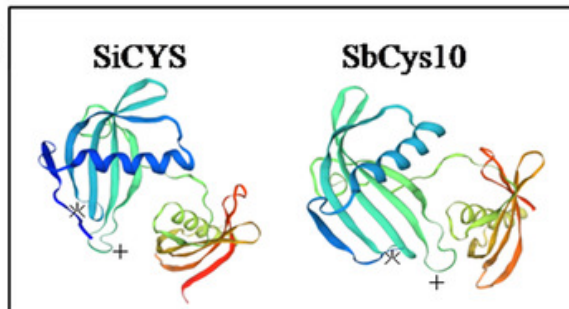




\section{Figure 5}

Phylogenetic relationships of the cystatins from Arabidopsis, rice, barley and Sorghum.

The phylogenetic tree was constructed by MEGA X with the maximum likelihood method. The numbers at the nodes indicate the bootstrap values. Gene names with black, red, and blue represented Group I, Group II, and Group III, respectively. 


\section{Group I}

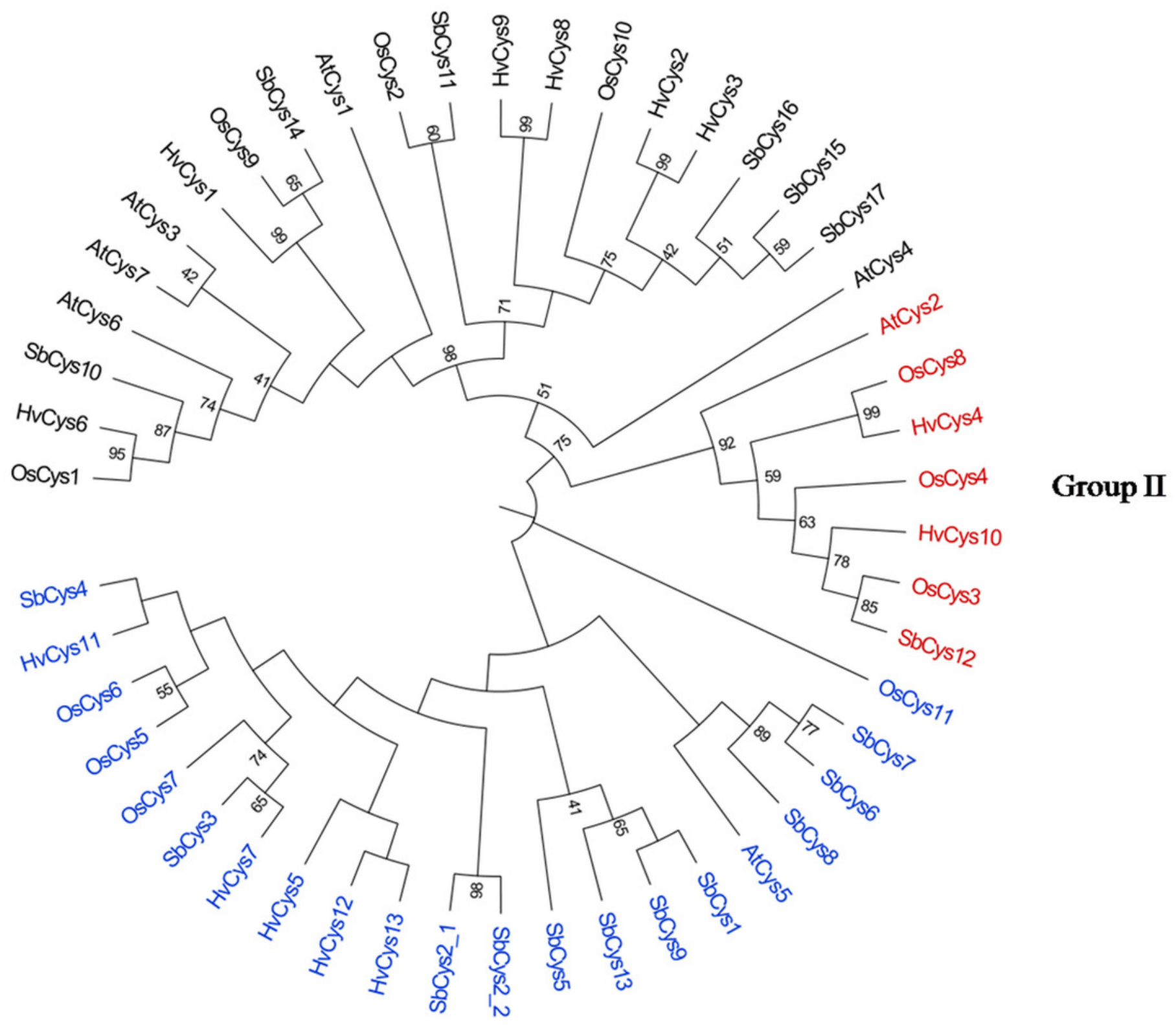

Group III 


\section{Figure 6}

The distribution of cis-elements in the $1.5 \mathrm{~kb}$ upstream promoter regions of SbCys genes.

The cis-elements in the promoter region of SbCys genes were predicted using PlantCARE database ( http://bioinformatics.psb.ugent.be/webtools/plantcare/html/ ). Different ciselements were represented by different shapes and colors.

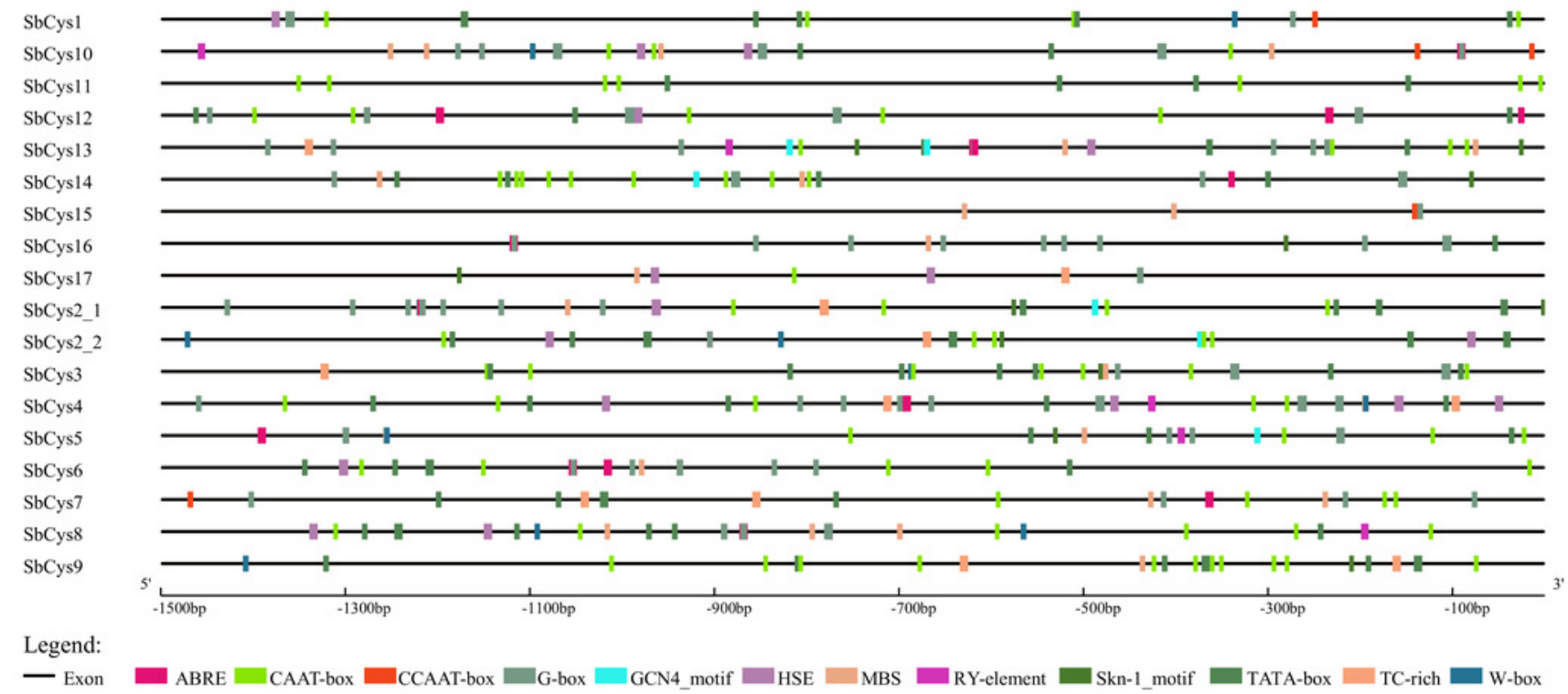




\section{Figure 7}

The interaction networks of SbCys proteins according to the orthologs in Arabidopsis.

Functional interacting network models were integrated using the STRING tool, and the confidence parameters were set at a 0.40 threshold. Homologous genes in Sorghum and Arabidopsis are shown in black and red, respectively.

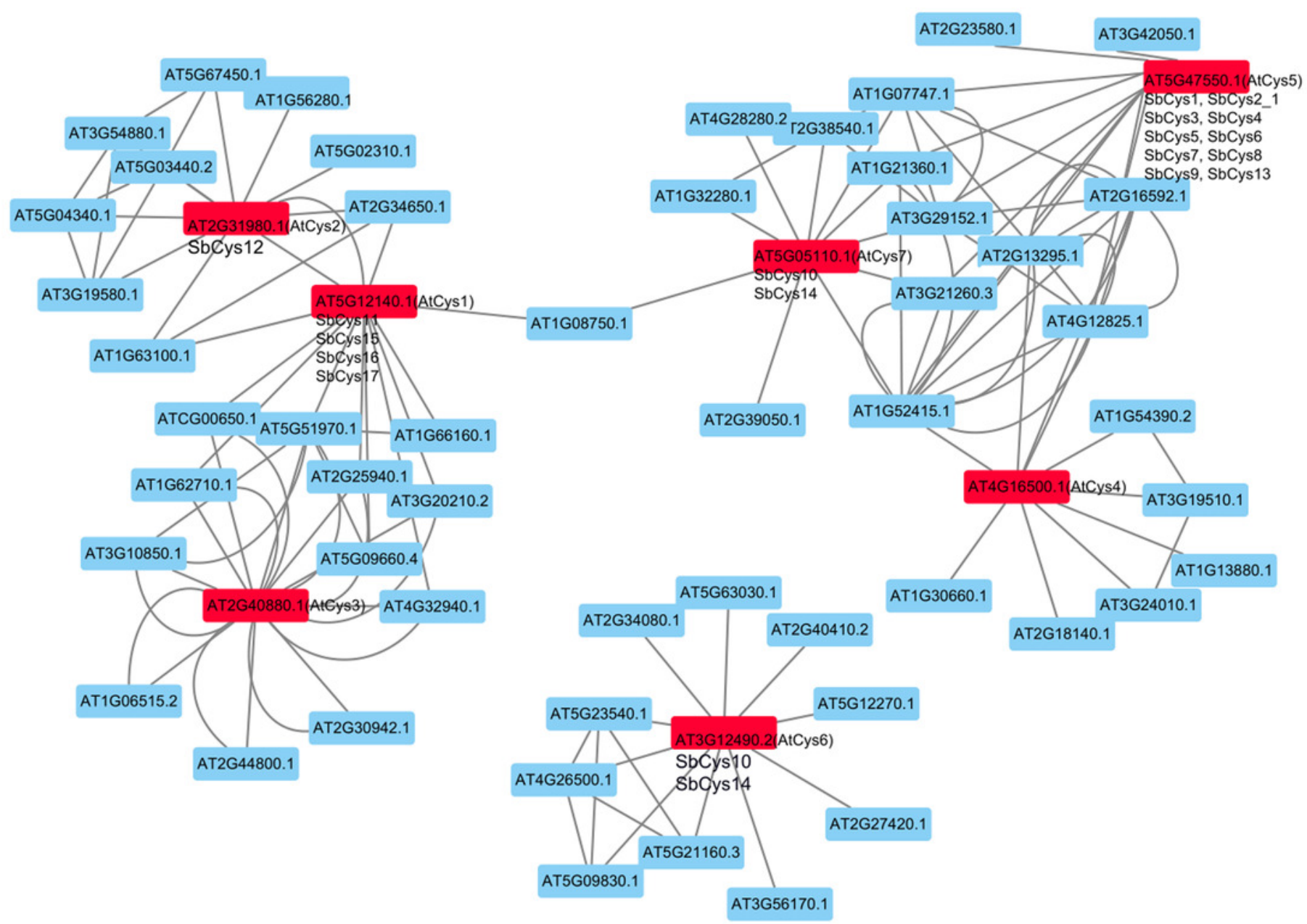




\section{Figure 8}

Hierarchical clustering of the expression profiles of SbCys genes in different tissues .

Different tissues are exhibited below each column. Root, shoot, and whole organism

belonged to vegetable tissues were collected at 14 days after Sorghum seed germination.

Reproductive tissues included embryo, endosperm and pericarp were collected at 20 days after pollination; pollens at booting stage; Inflorescences based on sizes: 1-5 mm, 5-10 mm, and 1-2 cm. Log transform data was used to create the heatmap. The scale bar represented the fold change (color figure online). Blue blocks represented the lower expression level and red blocks represented the higher expression level. 


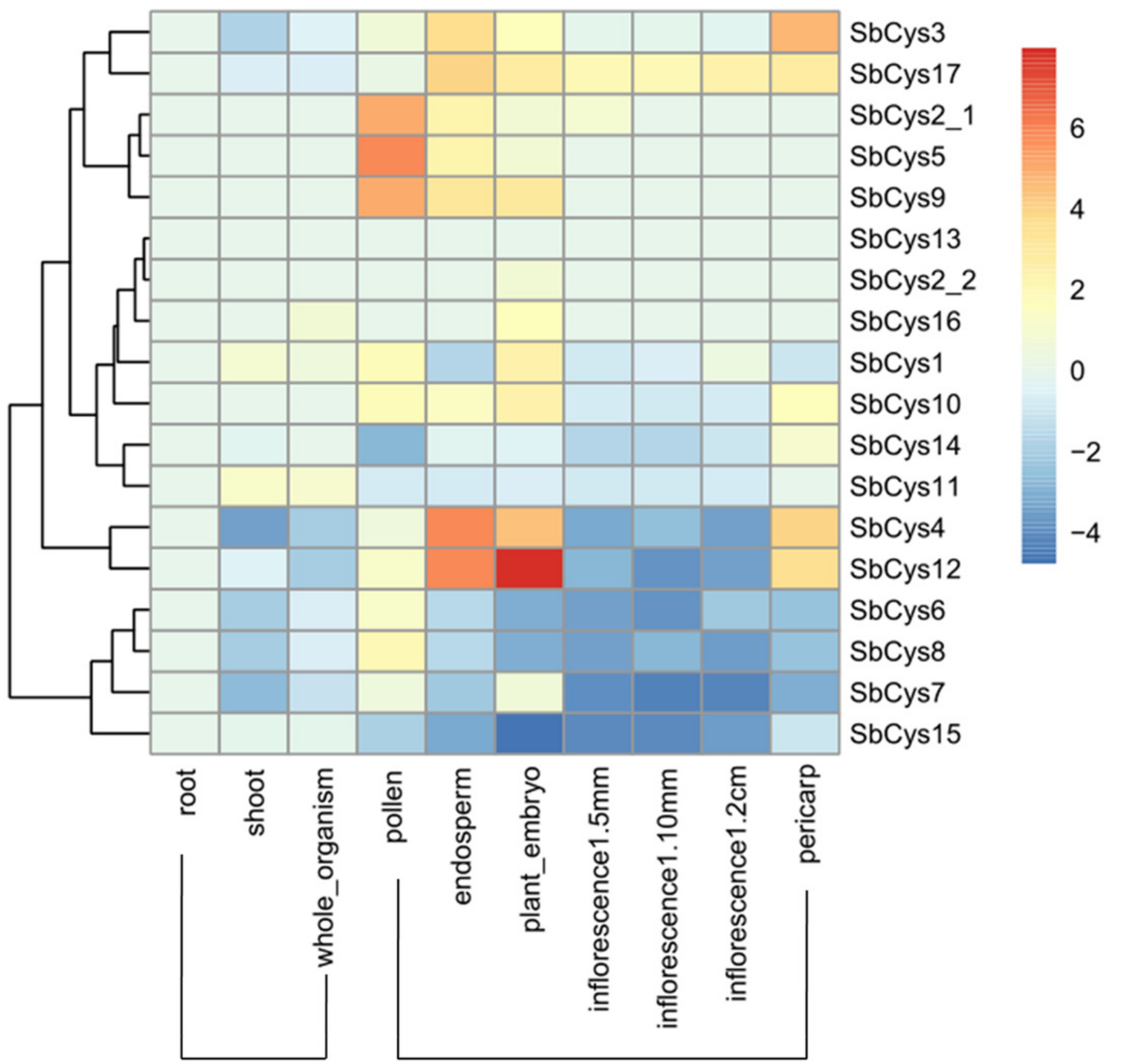

Vegetable tissues

Reproductive tissues 
Figure 9

Hierarchical clustering of the expression profiles of SbCys genes under biotic stresses.

(A) The expression changes in SbCys genes at 0, 12, and 24 hours with Bipolaris sorghicola infection. (B) The expression changes of $S b C y s$ genes at 5, 10, 15 days with sugarcane aphid infestation. Log transform data was used to create the heatmap. The scale bar represents the fold change (color figure online). Blue blocks indicate low expression and red blocks indicate high expression (color figure online).

A

B
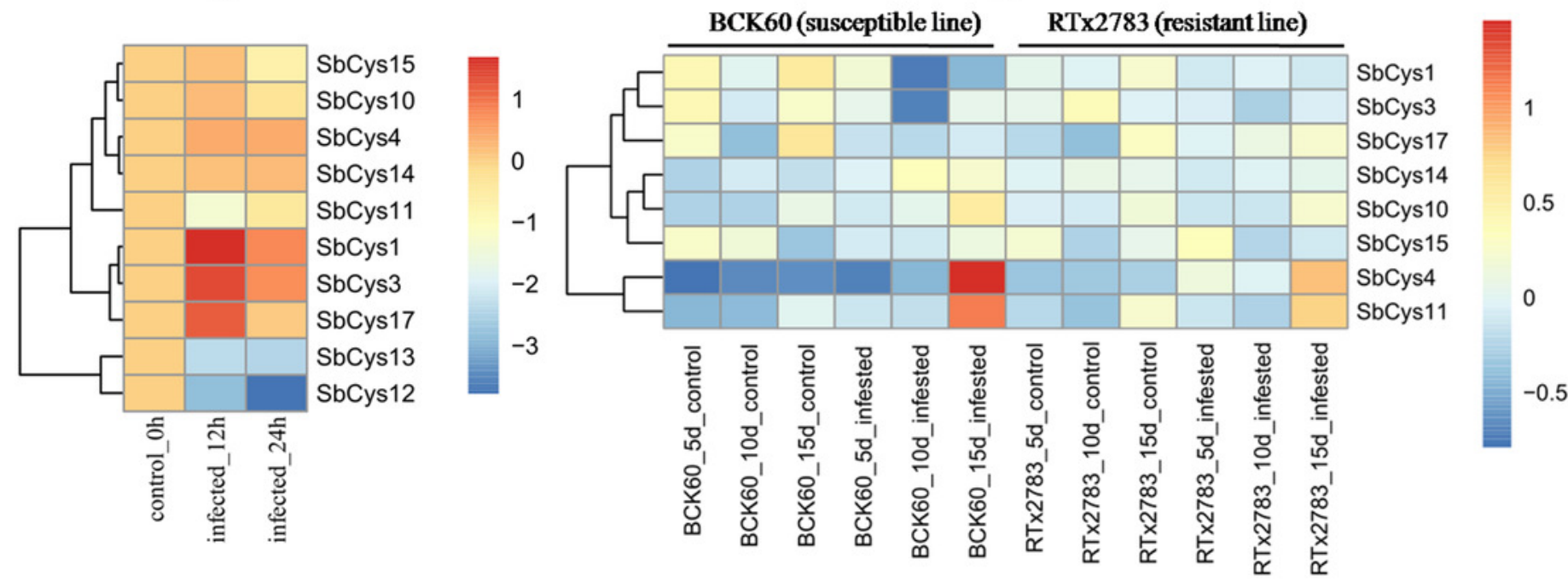
Figure 10

Expression profiles of SbCys genes at 5, 10, and 15 days with sugarcane aphid infection.

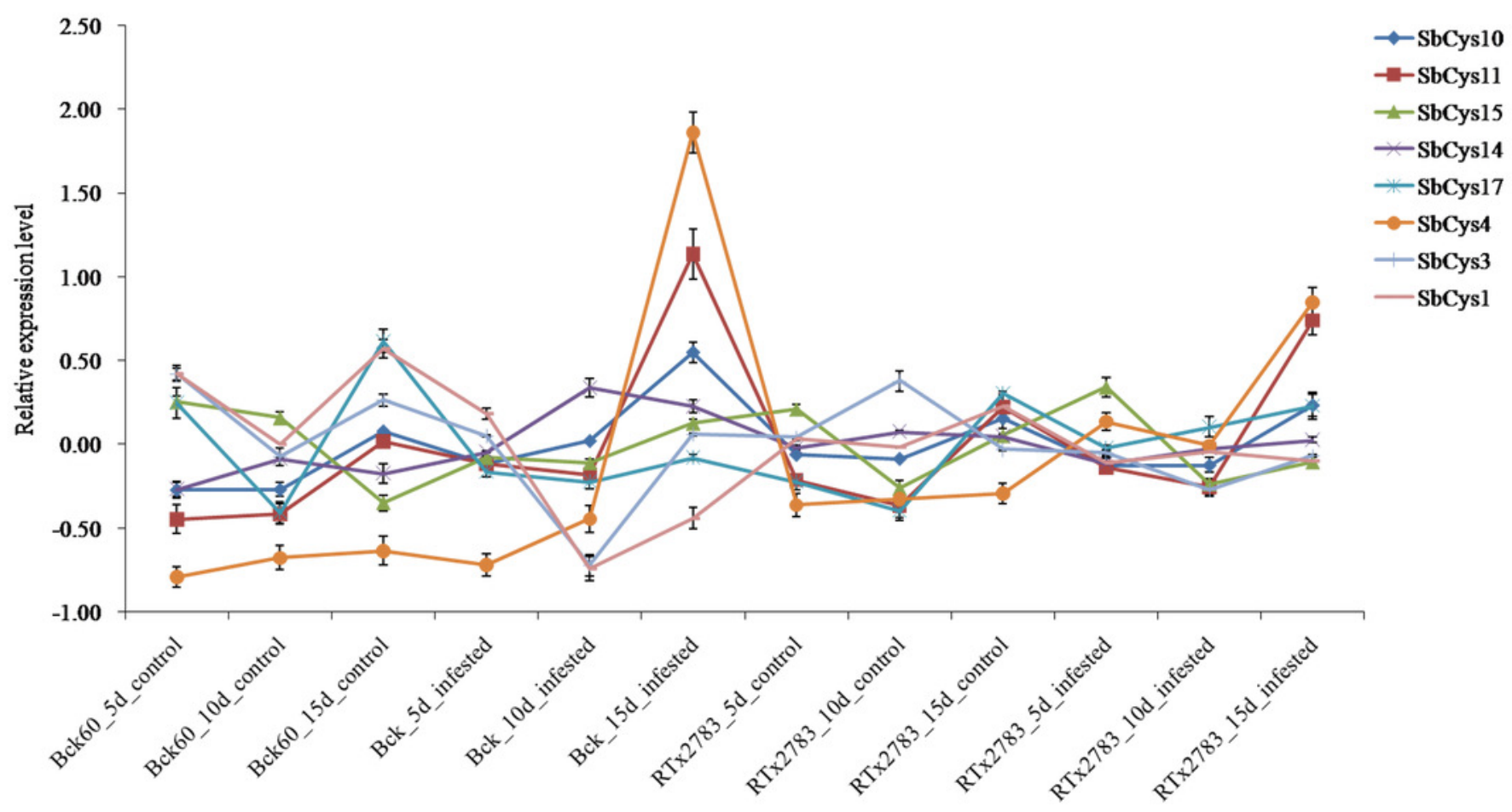


Figure 11

Expression patterns of SbCys genes under (A) dehydration (PEG 6,000) treatment, (B) salt shock $(\mathrm{NaCl})$ treatment, and $(\mathrm{C}) \mathrm{ABA}$ treatment.

qRT-PCR was used to investigate the expression levels of each SbCys gene. To visualize the relative expression levels data, $0 \mathrm{~h}$ at each treatment was normalized as " 1 ". * indicated significant differences in comparison with the control at $p \leq 0.05$. ** indicated significant differences in comparison with the control at $p \leq 0.01$. 

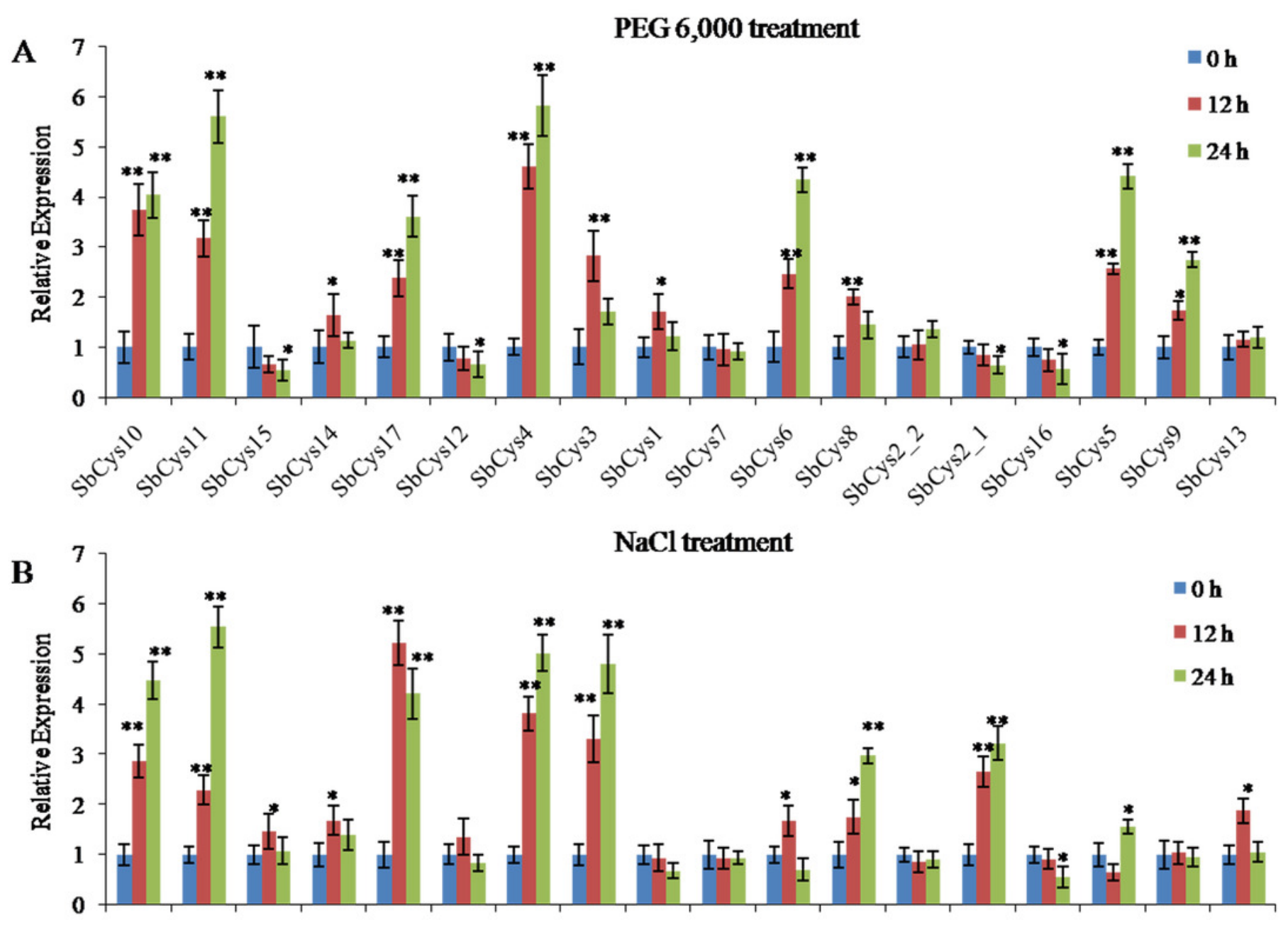

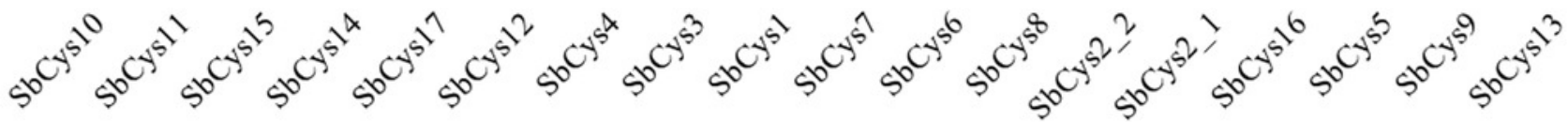

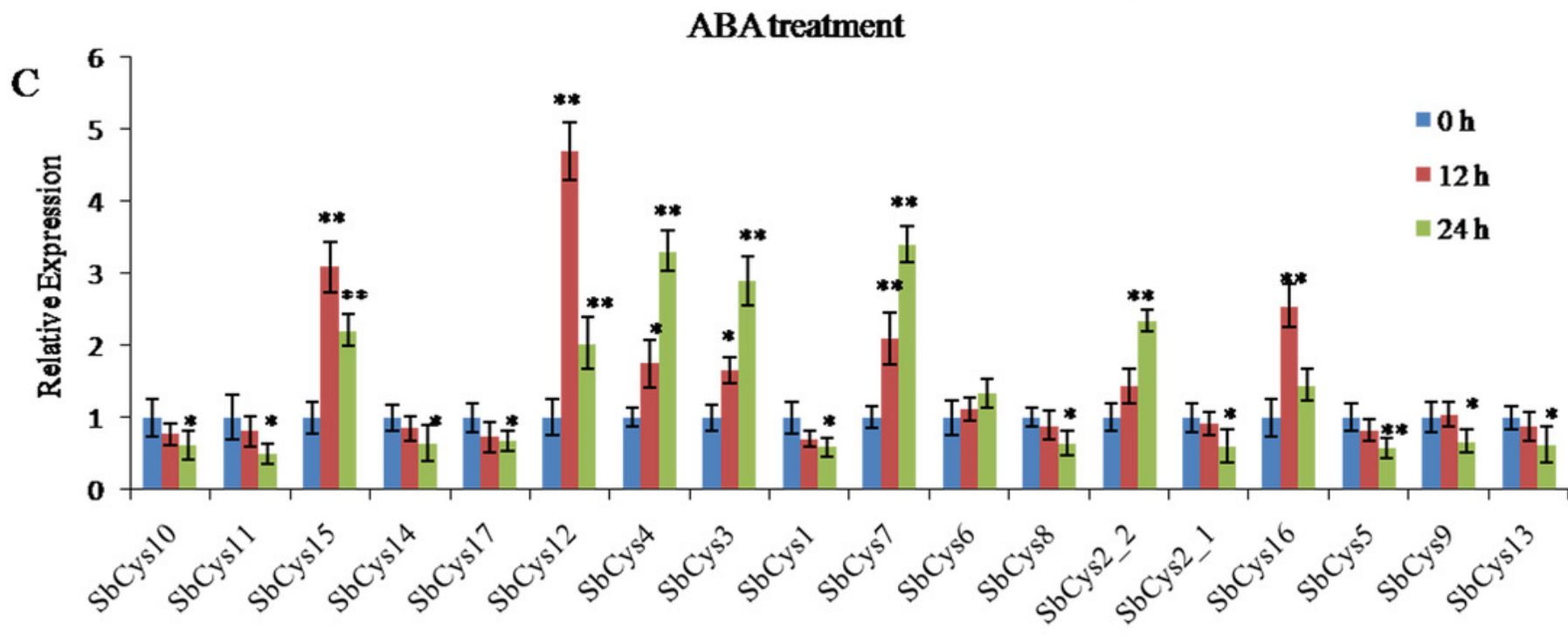

